\title{
Photoacoustic and thermoacoustic signal characteristics study
}

\author{
Nadaparambil Aravindakshan Rejesh, Manojit Pramanik ${ }^{*}$ \\ Biomedical Imaging Laboratory, Electrical Engineering, Indian Institute of Science, Bangalore, \\ India 560012
}

\begin{abstract}
Photoacoustic/thermoacoustic imaging is an emerging hybrid imaging modality combining optical/microwave imaging with ultrasound imaging. The photoacoustic/thermoacoustic signal generated are affected by the nature of excitation pulse waveform, pulse width, target object size, transducer size etc. In this study $k$-wave was used to simulate various configurations of excitation pulse, transducer types, and target object sizes and to see their effect on the photoacoustic/thermoacoustic signals. Numerical blood vessel phantom was also used to see the effect of various pulse waveform and excitation pulse width on the reconstructed images. This study will help in optimizing transducer design and reconstruction methods to obtain the superior reconstructed image.
\end{abstract}

Keywords: Photoacoustic, thermoacoustic, image reconstruction, $k$-wave

\section{INTRODUCTION}

Photoacoustic (PA) imaging - an emerging, noninvasive, biomedical imaging modality combining both optics and ultrasonic - allows in vivo visualization of embedded light absorbing structures ${ }^{1-4}$. In this technique, a biological tissue sample is illuminated with short pulses of laser light at a wavelength typically in the red or near-infrared (NIR) region. The absorption of light causes local heating which produces pressure waves, known as PA waves, via thermoelastic expansion. By using a wideband ultrasonic transducer one can acquire the PA waves that propagate back to the tissue surface. A single-element ultrasonic transducer or a transducer array is used to obtain the PA waves at various locations around the surface of the tissue. These PA waves are then used to reconstruct the initial pressure rise. Initial pressure rise is proportional to the absorbed optical energy, which is proportional to the light fluence distribution in the tissue and the absorption coefficient of the tissue. If a homogeneous fluence distribution is assumed then the initial pressure rise can be used to map the absorption coefficient of the tissue. A similar technology called thermoacoustic (TA) imaging, where instead of pulsed laser; a pulsed microwave source is used to irradiate the tissue $e^{5-9}$. Similar receiving mechanism is used to receive the TA waves and reconstruction is done to obtain microwave absorption map of the tissue instead of optical absorption.

A detailed understanding of the parameters that affect the PA/ TA signal, which will affect the reconstructed image, is essential. The factors which affect the PA/TA signals are the optical/microwave absorption properties of tissue, acoustic properties of the tissue, the excitation laser/microwave source characteristics (e.g., pulse width, pulse shape), absorber size and shape, characteristics of the ultrasound sensors (e.g., center frequency, bandwidth, element size and shape). Here, how these factors influence the generated PA/TA waves was studied. This will help in optimizing the detection of the signal and improving the reconstructed image.

Thermoacoustic signals generated by tumor targets with different sizes subject to microwave pulses with various widths and waveforms are theoretically investigated and impact of the pulse width on acoustic signal peak-to-peak interval and image spatial resolution was developed ${ }^{10}$. Generated time-domain thermoacoustic pressure is solved analytically and validity of the analytical approach was confirmed by finite-difference time-domain (FDTD) method ${ }^{11}$. Here, a k-space pseudo spectral solution method ${ }^{12}$ is used for the simulations. The main advantage of the numerical model used in $k$ Wave compared to models based on FDTD schemes is that fewer spatial and temporal grid points are needed for accurate simulations. This means the models run faster and use less memory. In this work, $k$-wave MATLAB Toolbox (MathWorks, Massachusetts, USA) was used for all the simulations.

*Email: manojit@ee.iisc.ernet.in; 


\section{METHODS}

$k$-Wave MATLAB toolbox was used to simulate PA/TA wave propagation in 3D. An absorbing boundary layer (in this case a perfectly matched layer, or PML) was used. Figure 1(A) shows the simulation geometry in 2D; however, all simulations were done in 3D. A computational grid of $210 \times 210 \times 210$ pixels (each pixel was $0.025 \mathrm{~mm}$ ) was used. The detector (red bar) was placed at a distance of $2 \mathrm{~mm}$ from the center of the target. Both point detector as well as singleelement large detector was used for simulations. The time step chosen was $10 \mathrm{~ns}$, and a total number of time steps were 728. The simulations assumed a sound speed of $1570 \mathrm{~m} / \mathrm{s}$. For simplicity the medium was considered homogeneous and there was no absorption or dispersion of sound. However, any other medium can also be considered. In all simulations the initial pressure rise was assumed to be $1 \mathrm{~Pa}$. Four types of excitation pulses: sinusoidal, square, gaussian and triangular were used. Figure 1(B) shows various pulse waveforms used for exciting the target with $4 \mu$ s pulse width. The rise time and fall time of the square pulse was chosen to be $t_{p} / 10$, where $t_{p}$ represents the pulse width. Although the figure shows only $4 \mu \mathrm{s}$ pulse width, simulations were done for various other pulse widths $(0.1,0.2,0.5,1,2$, and $4 \mu \mathrm{s})$ as well.
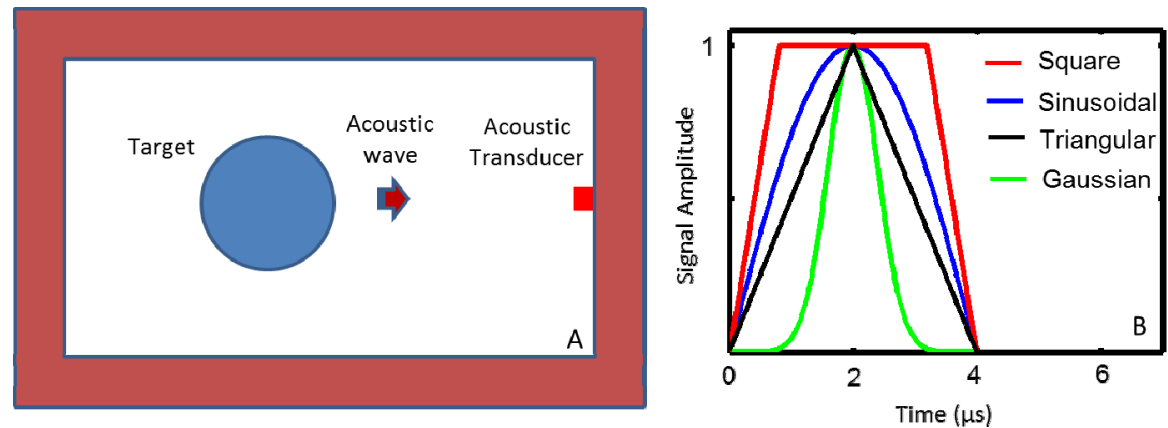

Figure 1: (A) Schematic diagram of the simulation geometry in 2D. However, actual simulations were done in 3D. (B) The excitation pulse waveforms: Sinusoidal, Square, $\mathrm{t}_{\mathrm{p}}$ (rise time and fall time of $\mathrm{t}_{\mathrm{p}} / 10$ ), Gaussian, and Triangular. In all the cases the pulse width $\left(\mathrm{t}_{\mathrm{p}}\right)$ was $4 \mu \mathrm{s}$.

\section{RESULTS AND DISCUSSION}

\subsection{Simulation with point detector}

The generated PA/TA pressure waves for sinusoidal, square, gaussian, and triangular pulses for various pulse widths $(0.1,0.2,0.5,1,2$, and $4 \mu \mathrm{s})$ are shown in Figures 2(A), 2(B), 2(C), and 2(D), respectively. Here the target radius was fixed at $1 \mathrm{~mm}$. As seen in Figure 2(A), the time duration of the generated PA/TA waves increased with the increase of excitation pulse width, indicating reduction the bandwidth of the signal. So for higher pulse width excitation, a low bandwidth transducer can be used for more efficient detection of the signal. The peak amplitude of the signal initially increased with the increase of pulse width, and decreased as pulse width goes beyond $1 \mu$ s. Similar results were obtained for other types of pulses, e.g., square pulse, gaussian pulse, and triangular pulse as shown in Figure 2(B), 2(C), and 2(D), respectively. When pulse width is large, the positive and negative lobes in the signal amplitude are separated for the square pulse case, as seen in Figure 2(B), which is obviously distinct from the other three waveforms. This is because, when the pulse waveform has a flat part, the time derivative is zero and thus makes no contribution to the initial pressure rise.

Figures 2(E), 2(F), 2(G), and 2(H) shows the PA/TA waves for sinusoidal, square, gaussian, and triangular excitation pulses (pulse width is fixed at $1 \mu \mathrm{s}$ ) for various target radius $(0.2,0.4,0.8$, and $1.6 \mathrm{~mm})$. The peak amplitude of the $\mathrm{PA} / \mathrm{TA}$ wave is slightly more when square excitation pulses were used. 

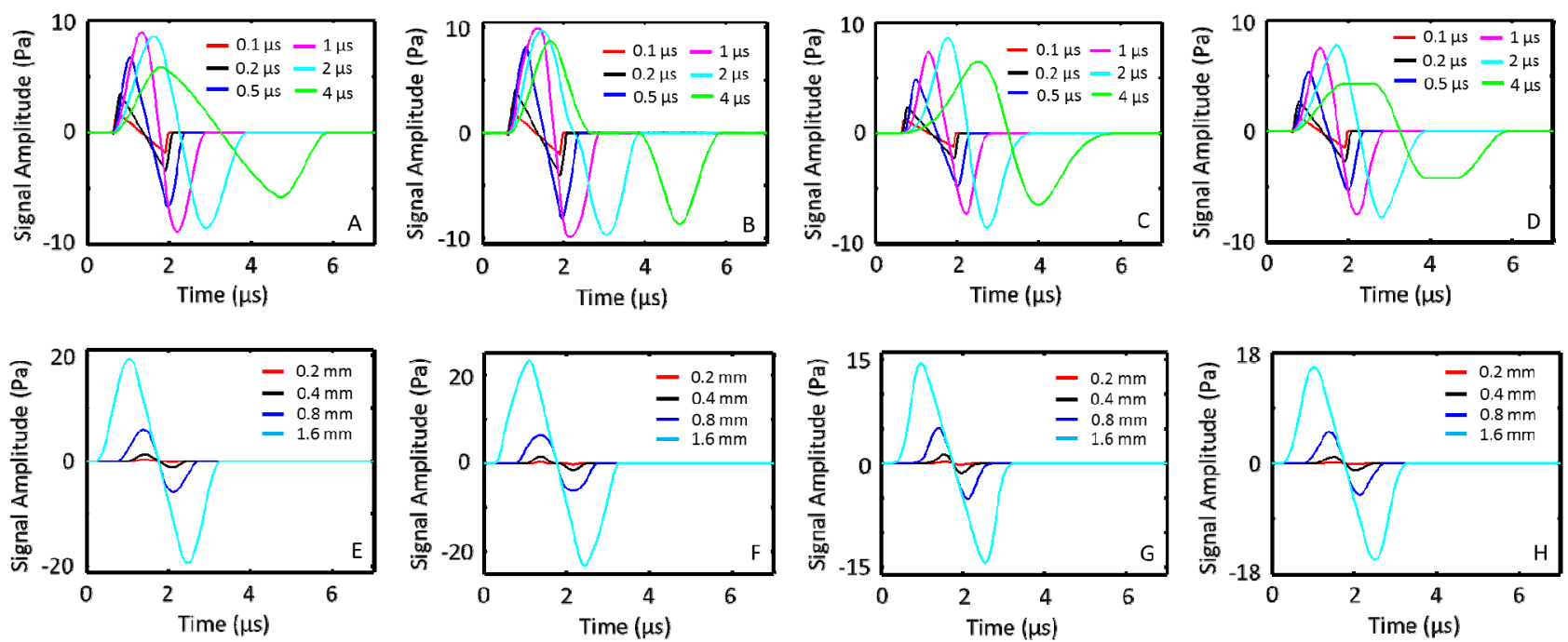

Figure 2: PA/TA waves generated from $1 \mathrm{~mm}$ radius spherical target with (A) sinusoidal, (B) square, (C) gaussian, and (D) triangular excitation pulse of varying pulse width. PA/TA waves generated from various spherical target size for (E) sinusoidal, $(\mathrm{F})$ square, $(\mathrm{G})$ gaussian, and $(\mathrm{H})$ triangular excitation pulse. The pulse width is fixed at $1 \mu$ s and the target was always located at the center of the computational grid. The transducer was assumed to be a point detector.

\subsection{Simulation with large detector}

The above simulations were repeated for a large nonfocused transducer of $6 \mathrm{~mm}$ diameter active area instead of a point detector, as in practice it is difficult to use a point detector due to its low sensitivity. Figure 3(A) shows similar results (as in the case of point detector, Figure 2(D)) obtained for a triangular pulse excitation for various pulse widths $(0.1,0.2$, $0.5,1,2$, and $4 \mu \mathrm{s}$ ) with large detector. Reduction in received signal bandwidth is observed due to the larger active area. Note that, when the pulse width is longer, the effect of broad transducer is not noticeable; however, when the pulse width is shorter, the broad active area of the transducer will reduce the effective bandwidth of the detected signal. Figure 3(B) shows similar results (similar to Figure $2(\mathrm{H})$ ) obtained from simulations using various target radius $(0.2,0.4,0.8$, and 1.6 $\mathrm{mm}$ ) with broad transducer.
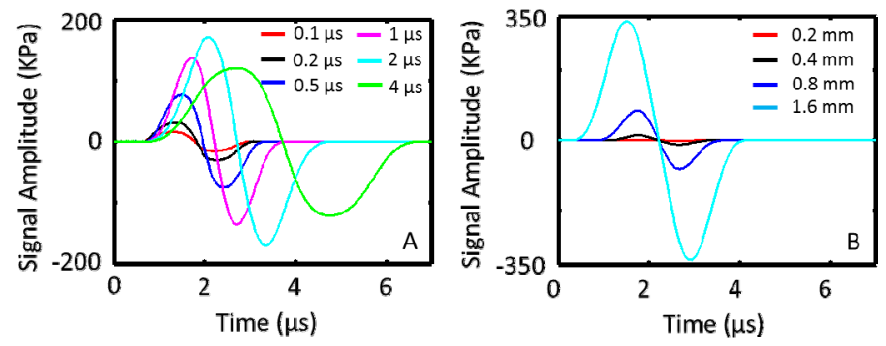

Figure 3: (A) PA/TA waves generated from $1 \mathrm{~mm}$ radius spherical target with triangular excitation pulse of varying pulse width with broad transducer (6 mm diameter active area transducer), similar to Figure 2(D). (B) PA/TA waves generated with $1 \mu$ s triangular excitation pulse with various spherical target size with broad transducer, similar to Figure $2(\mathrm{H})$.

\subsection{Spectrum of PA/TA waves with point detector}

The frequency domain profile for the PA/TA waves generated from sinusoidal, square, gaussian, and triangular pulses for different pulse widths is shown in Figure 4(A), 4(B), 4(C), and 4(D), respectively. The spectral magnitude was normalized for different pulse widths. A reduction in the side lobes were seen for all input pulses as the pulse width increased. The peak frequency of the main lobe decreases for all inputs as the pulse width increased. Figures 4(E), 4(F), $4(\mathrm{G})$, and $4(\mathrm{H})$ shows the spectrum of PA/TA waves for various target sizes with sinusoidal, square, gaussian, and triangular pulse inputs, respectively. The side lobe reduction was observed for larger targets. 

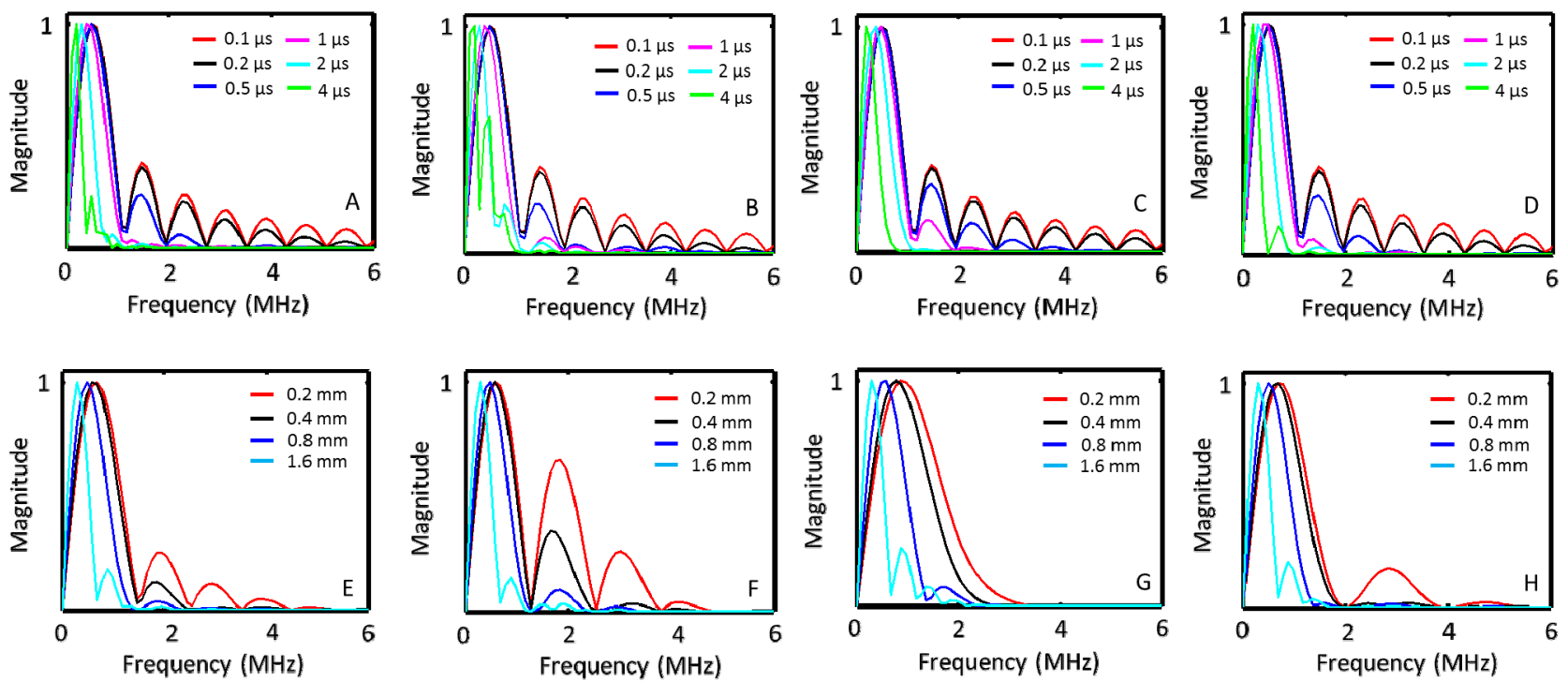

Figure 4: Spectrum of PA/TA waves generated from $1 \mathrm{~mm}$ radius spherical target with (A) sinusoidal, (B) square, (C) gaussian, and (D) triangular excitation pulse of varying pulse width. Spectrum of PA/TA waves generated from various spherical target size for $1 \mu \mathrm{s}(\mathrm{E})$ sinusoidal, $(\mathrm{F})$ square, $(\mathrm{G})$ gaussian, and $(\mathrm{H})$ triangular excitation pulse. The target was always located at the center of the computational grid. The transducer was assumed to be a point detector.

\subsection{Spectrum of PA/TA waves with large detector}

Figure 5(A) shows frequency domain profile of the detected PA/TA signals obtained with a triangular pulse excitation for various pulse widths $(0.1,0.2,0.5,1,2$, and $4 \mu \mathrm{s})$ using the broad transducer instead of a point detector. Note that, the numbers of side lobes were reduced in comparison with the point detector. Figure 5(B) shows similar results obtained from simulations using various target radius $(0.2,0.4,0.8$, and $1.6 \mathrm{~mm})$ for a fixed excitation pulse width.
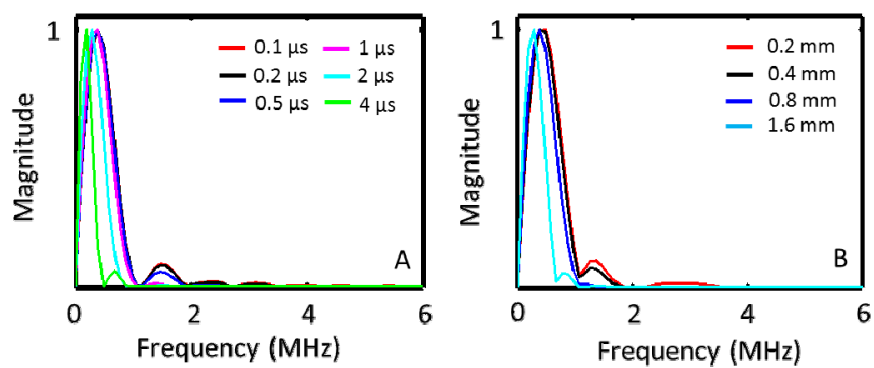

Figure 5: (A) Spectrum of PA/TA waves generated from $1 \mathrm{~mm}$ radius spherical target with triangular excitation pulse of varying pulse width with broad transducer. (B) Spectrum of PA/TA waves generated with $1 \mu$ s triangular excitation pulse with various spherical target size with broad transducer. The target was always located at the center of the computational grid.

\section{IMAGE RECONSTRUCTION}

All the simulations done till now were to study the generated PA/TA waveforms. Next, numerical phantoms were taken and image reconstructions were done to map the initial pressure rise. Figure 6(A) shows the numerical blood vessel phantom used. The initial pressure rise was assumed to be $1 \mathrm{~Pa}$. The simulations were carried out in $2 \mathrm{D}$ to reduce computational complexity. A computational grid of 401 x 401 x 401 pixels (each pixel was $0.1 \mathrm{~mm}$ ) was used. 200 
detectors were placed at a radius of $18 \mathrm{~mm}$ from the centre of the computational grid to collect data. Once again $k$-wave was used to generate the forward simulated data as well as for the time-reversal reconstruction.

Sound speed in the medium was assumed to be $1500 \mathrm{~m} / \mathrm{s}$. The time step chosen was $50 \mathrm{~ns}$, and a total number of time steps were 600 . The forward data was generated by giving a gaussian pulse excitation for various pulse widths $(0.1 \mu \mathrm{s}$, $0.25 \mu \mathrm{s}, 0.5 \mu \mathrm{s}$, and $1 \mu \mathrm{s})$. A $40 \mathrm{~dB}$ gaussian noise was added to the sensor data. The photoacoustic image reconstruction was done using the time varying pressure recorded over all the detectors. Individual detectors were assumed to be point detector.
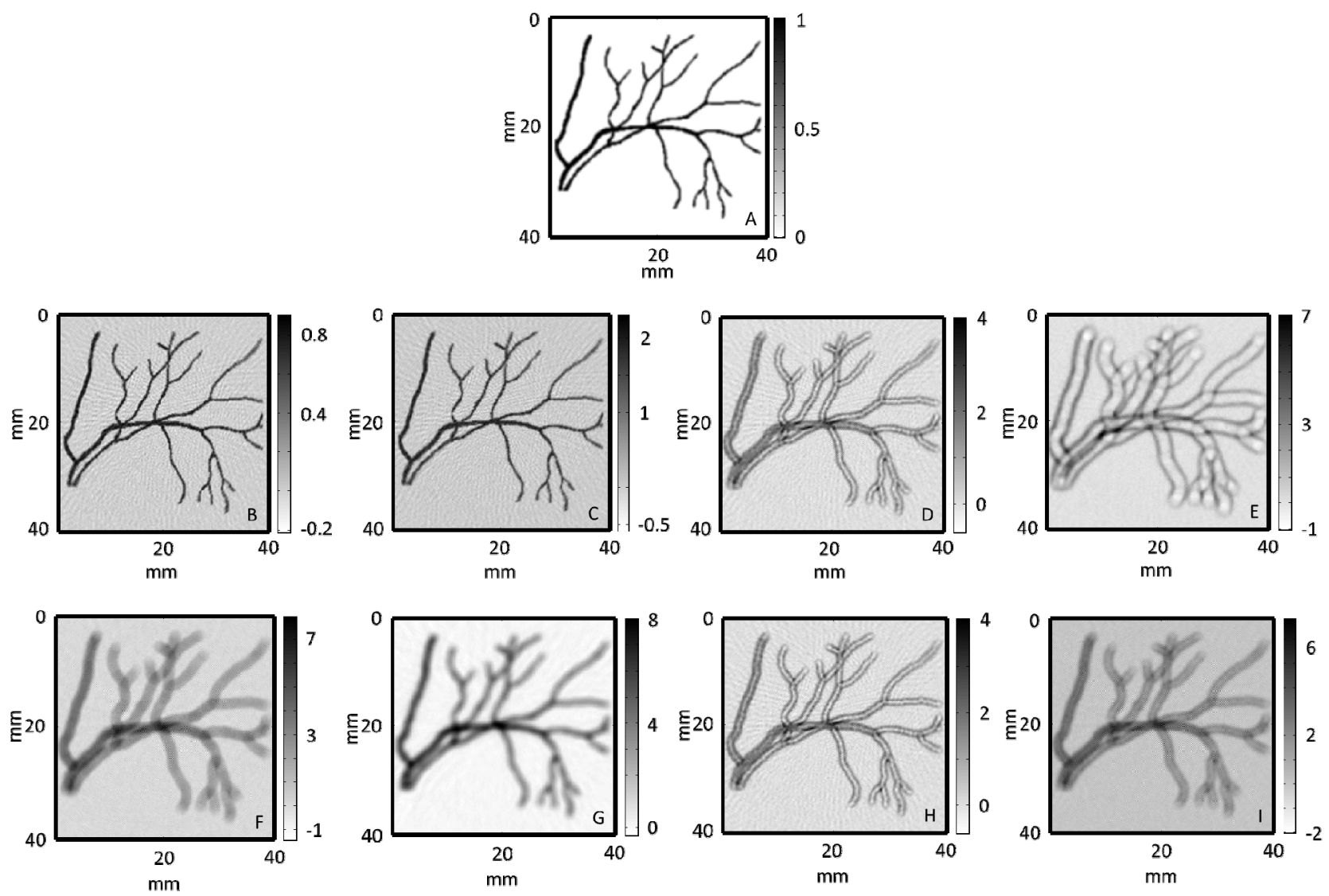

Figure 6: (A) Numerical blood vessel phantom. Initial pressure rise assumed to be $1 \mathrm{~Pa}$. Reconstructed image for a gaussian excitation with various pulse width (B) $0.1 \mu \mathrm{s}$, (C) $0.25 \mu \mathrm{s}$, (D) $0.5 \mu \mathrm{s}$, and (E) $1 \mu \mathrm{s}$. Reconstructed images for various excitation waveform with $0.5 \mu$ s pulse width $(\mathrm{F})$ sinusoidal, $(\mathrm{G})$ square, $(\mathrm{H})$ gaussian, and $(\mathrm{I})$ triangular.

The reconstructed images for a gaussian pulse input with various pulse widths $(0.1 \mu \mathrm{s}, 0.25 \mu \mathrm{s}, 0.5 \mu \mathrm{s}$, and $1 \mu \mathrm{s})$ is shown in Figure 6(B), 6(C), 6(D), and 6(E), respectively. As expected the reconstructed image quality degrades for larger pulse widths. With sinusoidal, square, gaussian and triangular excitation pulses the reconstructed images are shown in Figure 6(F), 6(G), 6(H), and 6(I), respectively. The pulse width was $0.5 \mu$ s. Better reconstructed images were obtained for square pulse excitation. One way of improving the reconstructed images is using deconvolution. Mathematically, the reconstructed image is degraded due to the convolution of the transfer function of the imaging system with the excitation pulse waveform. So if the pulse waveform shape is known it is possible to recover the exact images by deconvolution. However, deconvolution method is sometimes corrupted by the noise. In the future, we will be working on improving the image quality by deconvolution method. 


\section{CONCLUSION}

In this work we have tried to explore the effect of different excitation pulse waveforms of different pulse width and different target sizes on the generated PA/TA waves. It is shown that for higher pulse width excitation reduces the bandwidth of the generated signals. Also the surface area of the detectors plays a role in the detected signals. Theoretically, one can calculate the generated signals using analytical methods, however, with complicated geometry; inhomogeneous medium analytical solutions may not exist. Therefore, numerical methods like $k$-wave based solution plays an important role in understanding the signal behavior for different imaging configuration. Image reconstruction with numerical phantoms was also looked into using $k$-wave time reversal reconstruction. Square pulse excitation gave better results. We are trying to improve the image quality using deconvolution methods. In the future, we will extend this work for more complicated, inhomogeneous medium.

\section{REFERENCES}

[1] M. H. Xu and L. V. Wang, "Photoacoustic imaging in biomedicine,"RevSci. Instrum. 77, 041101 (2006).

[2] Da X. Wang, Y. Pang, G. Ku, X. Xie, G. Stoica, and L.H. V. Wang, "Noninvasive laser-induced photoacoustic tomography for structural and functional in vivo imaging of the brain," Nature Biotechnology 21, 803-806, (2003).

[3] M. Pramanik, G. Ku, C. H. Li, and L. H. V. Wang, "Design and evaluation of a novel breast cancer detection system combining both thermoacoustic (TA) and photoacoustic (PA) tomography," Med.Phys. 356, 2218 2223(2008).

[4] C. G. A. Hoelen, F. F. M. de Mul, R. Pongers, and A. Dekker, "Three dimensional photoacoustic imaging of blood vessels in tissue," Opt Lett. 238, 648-650 (1998)

[5] L. H. V. Wang, X. M. Zhao, H. T. Sun, and G. Ku, "Microwave-induced acoustic imaging of biological tissues," Rev. Sci. Instrum. 70, 3744-3748 (1999).

[6] M. H. Xu and L. H. V. Wang, "Pulsed-microwave-induced thermoacoustic tomography: Filtered backprojection in a circular measurement configuration," Med. Phys. 29, 1661-1669 (2002).

[7] L. V. Wang, X. M. Zhao, H. T. Sun, and G. Ku, "Microwave-induced acoustic imaging of biological tissues,"Rev. Sci. Instrum.70, 3744- 3748 (1999).

[8] Lawrence E. Larsen and John H. Jacobi., [Medical Applications of Microwave Imaging], IEEE Press, New York, 1986.

[9] G. Ku and L. H. V. Wang, "Scanning microwave-induced thermoacoustic tomography: signal, resolution, and contrast," Med. Phys. 27, 1195-1202 (2000).

[10]X. Wang, D. R. Bauer, J. L. Vollin, D. G. Manzi, R. S. Wittie, and H. Xin, "Impact of Microwave pulses on Thermoacoustic Imaging Applications", IEEE Antennas and Wireless Propagation Letters 11, 1634 (2012).

[11]C. Lou, L. Nie, and D. Xu, "Effect of excitation pulse on thermoacoustic signal characteristics and the corresponding algorithm for optimization of image resolution", J.Appl. Phys. 110, 083101 (2011).

[12]B. E. Treeby and B. T. Cox, "k-Wave: MATLAB toolbox for the simulation and reconstruction of photoacoustic wave-fields," J. Biomed. Opt. 15, 021314 (2010).

[13]K. G. Zhu and M. Popovic, "Spectral difference between microwave radar and microwave-induced thermoacoustic signals," IEEE Antennas Wireless Propag. Lett., 8, 1259-1262( 2009).

[14] M. Xu and L. V. Wang, "Time-domain reconstruction for thermoacoustic tomography in a spherical geometry," IEEE Trans. Med. Imag.,21, 814-822, (2002). 